\title{
Luminescence properties of transition-metal-doped GaSb
}

\author{
P. Hidalgo and B. Méndez \\ Departamento de Física de Materiales, Facultad de Físicas, Universidad Complutense, E-28040 Madrid, Spain \\ P. S. Dutta \\ Department of Mechanical Engineering, Rensselaer Polytechnic Institute, Troy, New York 12180 \\ J. Piqueras \\ Departamento de Física de Materiales, Facultad de Físicas, Universidad Complutense, E-28040 Madrid, Spain \\ E. Dieguez \\ Departamento de Física de Materiales, Universidad Autónoma de Madrid, 28049 Madrid, Spain
}

(Received 15 July 1997; revised manuscript received 15 September 1997)

\begin{abstract}
The luminescence properties of transition-metal $(\mathrm{Cr}, \mathrm{V}$, and $\mathrm{Ru}$ )-doped $\mathrm{GaSb}$ single crystals have been studied by a cathodoluminescence (CL) technique in a scanning electron microscope. Spatial segregation of impurities along the longitudinal direction of the crystals grown by the Bridgman method has been investigated. These dopants suppress the native acceptor concentration to varying extent. The behavior of Ru has been found to be different from the behavior of $\mathrm{V}$ and $\mathrm{Cr}$. In particular the complete disappearance of the $777 \mathrm{meV}$ (band A) emission has been observed in GaSb:Ru with a low doping level. A peak at $767 \mathrm{meV}$ is also seen in this sample which, to the best of our knowledge, has not been previously observed in GaSb. The CL results have been complemented by $\mathrm{x}$-ray-microanalysis measurements to ascertain the effect of doping level on the luminescence properties. [S0163-1829(98)05608-2]
\end{abstract}

\section{INTRODUCTION}

The transition group metals have a common feature: partial occupancy of the $d$ shells. In III-V compounds, this gives rise to several important technological applications and in particular, production of high resistivity materials through transition-metal doping has been widely used. It is surprising to see that there has been an enormous amount of work on transition metals in all III-V compounds except GaSb. ${ }^{1,2} \mathrm{Un}$ til now the only detailed work on transition metals in $\mathrm{GaSb}$ was on manganese. ${ }^{3,4} \mathrm{GaSb}$ is emerging as a potential substrate material for various lattice-matched devices operating in the $0.3-1.6 \mathrm{eV}$ range. ${ }^{5}$ Doping of $\mathrm{GaSb}$ has been studied by a few groups, however, its study is still in its infancy. However, significant progress needs to be made to completely understand the behavior of impurities in GaSb. Asgrown $\mathrm{GaSb}$ is always $p$ type in nature due to the native acceptors, which are gallium vacancies and gallium on antimony sites (antisites). Reducing the native acceptor concentration has been the challenge of the last three decades. The main method of reducing the native acceptor concentration has been to employ low-growth temperatures in epitaxial techniques.

In the present work, we investigate the effect of transition metals (chromium, vanadium, ruthenium) on the native acceptor concentration. The donor or acceptor nature of the transition metals is difficult to ascertain. The transition metals will form either deep donor levels or deep acceptor levels which will trap charge carriers and give rise to high resistivity as in other III-V compounds. As we have mentioned there is a lack of data regarding the behavior of transition metals in GaSb. For defect characterization, cathodoluminescence
(CL) in the scanning electron microscope (SEM) has been extensively applied to many semiconductors and semiconductor heterostructures. This technique which provides information on the nature and spatial distribution of the defects has been to our knowledge, only recently applied to characterize $\mathrm{GaSb}$ crystals as reported in previous works of our laboratory. 6,7

\section{EXPERIMENT}

The samples studied in this work were GaSb:Cr, GaSb:V, and GaSb:Ru crystals grown by the vertical Bridgmann

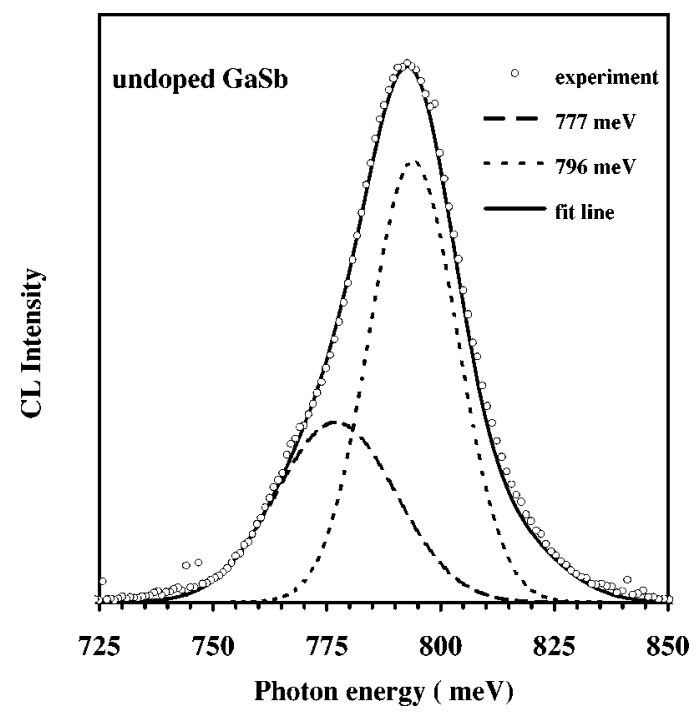

FIG. 1. CL spectrum of an undoped reference GaSb sample grown by the vertical Bridgman technique. 
TABLE I. Positions peaks at $77 \mathrm{~K}$ obtained from CL spectra in the different samples studied in this work. Data are in meV.

\section{$\mathrm{GaSb}: \mathrm{Cr}$}

\begin{tabular}{cccc} 
sample Cr1 & sample Cr2 & sample Cr3 & sample Cr4 \\
\hline 795 & 798 & 796 & 800 \\
770 & 777 & 775 & 777
\end{tabular}

GaSb:V

\begin{tabular}{cccc} 
sample V1 & sample V2 & sample V3 & sample V4 \\
\hline 807 & 807 & 801 & 795 \\
& & 778 & 775
\end{tabular}

$\mathrm{GaSb}: \mathrm{Ru}$

\begin{tabular}{cccc} 
sample Ru1 & sample Ru2 & sample Ru3 & sample Ru4 \\
\hline 777 & 793 & 792 & 790 \\
768 & 767 & 767 & 784 \\
\hline \hline
\end{tabular}

method by tip nucleation without a seed (see Ref. 5). The doping of the crystals was done by adding the respective metals to the melt at the level of $10^{19} \mathrm{~cm}^{-3}$. There is no solubility data for these metals in GaSb. Due to the preliminary nature of the work, the solubility was not investigated in any detail. The crystals were $12 \mathrm{~mm}$ in diameter and 40-50 $\mathrm{mm}$ in length. After the growth, from each crystal four disks were cut at various places along the length of the ingot and the wafers were chemomechanically polished to a mirror finish. Spatial and spectral CL measurements were carried out on these wafers. The samples are labeled with the symbol of the dopant metal $(\mathrm{Cr}, \mathrm{V}, \mathrm{Ru})$ followed by a number from 1 to 4 along the growth direction, corresponding to the bottom and to the top of the crystal.

The CL measurements were carried out using a Hitachi S-2500 scanning electron microscope at $77 \mathrm{~K}$ and at accelerating voltages of $20-30 \mathrm{kV}$. The details of the experimental setup for spectral and panchromatic CL measurements are

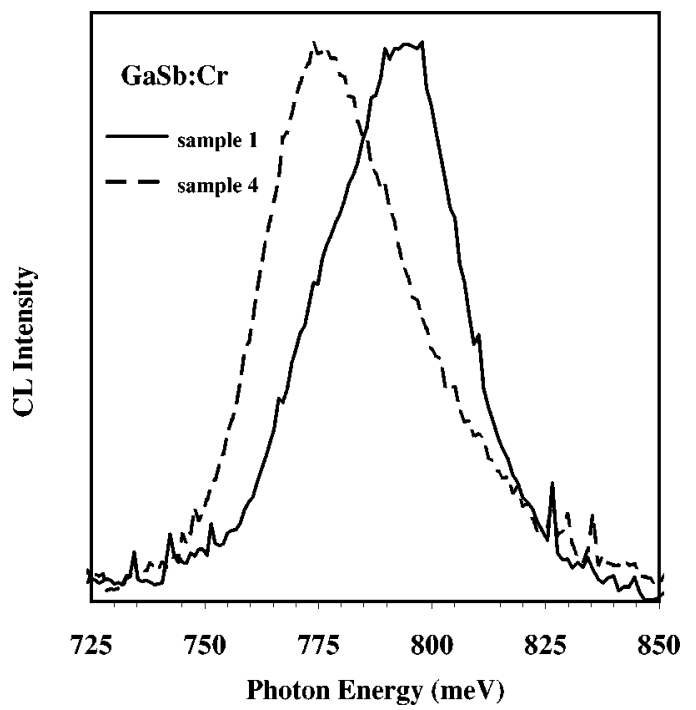

FIG. 2. Evolution of CL spectra in $\mathrm{GaSb}: \mathrm{Cr}$ along the growth direction. presented elsewhere. ${ }^{8}$ In order to study the composition of some of the features observed in the SEM micrographs, mapping of the elements $\mathrm{Ga}, \mathrm{Sb}, \mathrm{Cr}, \mathrm{V}$, and $\mathrm{Ru}$ was done by energy dispersive $\mathrm{x}$-ray microanalysis in a Jeol JXA-8900 M superprobe.

\section{RESULTS AND DISCUSSION}

The CL spectrum of an undoped GaSb sample is shown in Fig. 1. The dominant transitions for the undoped sample take place at 777 and $796 \mathrm{meV}$. Undoped GaSb is $p$ type in nature with intrinsic acceptors formed by gallium vacancies $\left(\mathrm{V}_{\mathrm{Ga}}\right)$ and gallium antisites $\left(\mathrm{Ga}_{\mathrm{Sb}}\right)$. These acceptors induce a luminescence band at $777 \mathrm{meV}^{\mathrm{m}}$ usually called ${ }^{9}$ band $\mathrm{A}$. The $796 \mathrm{meV}$ band corresponds to the band to band transition at $77 \mathrm{~K}$. It has been found that GaSb grown from Sb-rich melts has a lower concentration of native acceptors than $\mathrm{GaSb}$ grown from stoichiometric or Ga-rich melts, leading to a decrease of the $777 \mathrm{meV}$ peak in photoluminescence (PL) spectra. ${ }^{10}$ The luminescence from the doped samples is described in the following sections. In order to compare the obtained results we present in Table I the CL emission bands detected in the different doped samples. These transitions have been determined from the best fits to experimental CL spectra using a sum of Gaussian line distributions obtained by the Jandel Scientific PEAKFIT program.

\section{A. GaSb:Cr}

Our previous investigations of $\mathrm{GaSb}: \mathrm{Cr}$ samples showed the existence of impurity segregation during the growth process in the Bridgman method. ${ }^{11}$ The segregation coefficient is lower than unity in these samples, leading to higher $\mathrm{Cr}$ concentration in the sample labeled $\mathrm{Cr} 1$ and the lowest concentration in sample $\mathrm{Cr} 4$.

The incorporation of $\mathrm{Cr}$ affects the native defects in $\mathrm{GaSb}$ in the following way: native defect creation is believed to be initiated during the growth process by the volatilization of $\mathrm{Sb}$ with formation of $\mathrm{Sb}$ vacancies. This leads in a further step to the formation of Ga-antisites according to the scheme $\mathrm{Ga}_{\mathrm{Ga}}+\mathrm{V}_{\mathrm{Sb}} \rightarrow \mathrm{Ga}_{\mathrm{Sb}}+\mathrm{V}_{\mathrm{Ga}}$. With the incorporation of $\mathrm{Cr}$, however, the complex $\mathrm{Cr}-\mathrm{V}_{\mathrm{Sb}}$ is formed similar to the complex $\mathrm{Mn}-\mathrm{V}_{\mathrm{Sb}}$ in GaSb:Mn, which has been observed by photoluminescence and electron spin resonance. ${ }^{12}$ According to these assumptions, it is possible to reduce the native defect concentration by doping with Chromium. Our results confirm these suggestions. We observe that the intensity of the native acceptor band $\mathrm{A}$ increases along the growth direction with decreasing $\mathrm{Cr}$ content. Figure 2 shows the evolution of CL spectra from sample $\mathrm{Cr} 1$ to $\mathrm{Cr} 4$ in $\mathrm{GaSb}$ :Cr. The band A is weak or absent in the sample $\mathrm{Cr} 1$, where the $\mathrm{Cr}$ concentration is highest, and is dominant in sample $\mathrm{Cr} 4$, where the $\mathrm{Cr}$ concentration is lowest. The $796 \mathrm{meV}$ CL band corresponds to near band edge recombination at $77 \mathrm{~K}$. Evolution of the CL spectra with increasing temperature in these samples shows a shift to lower energies of the band A peak position, as well as of the fundamental emission.

The different degree of the incorporation of $\mathrm{Cr}$ along the growth direction, is reflected in the CL images obtained from the different samples. In the sample $\mathrm{Cr} 4$, the $\mathrm{CL}$ image shows a contrast which resembles the CL contrast in undoped samples, i.e., dark contrast of the subgrain boundaries 

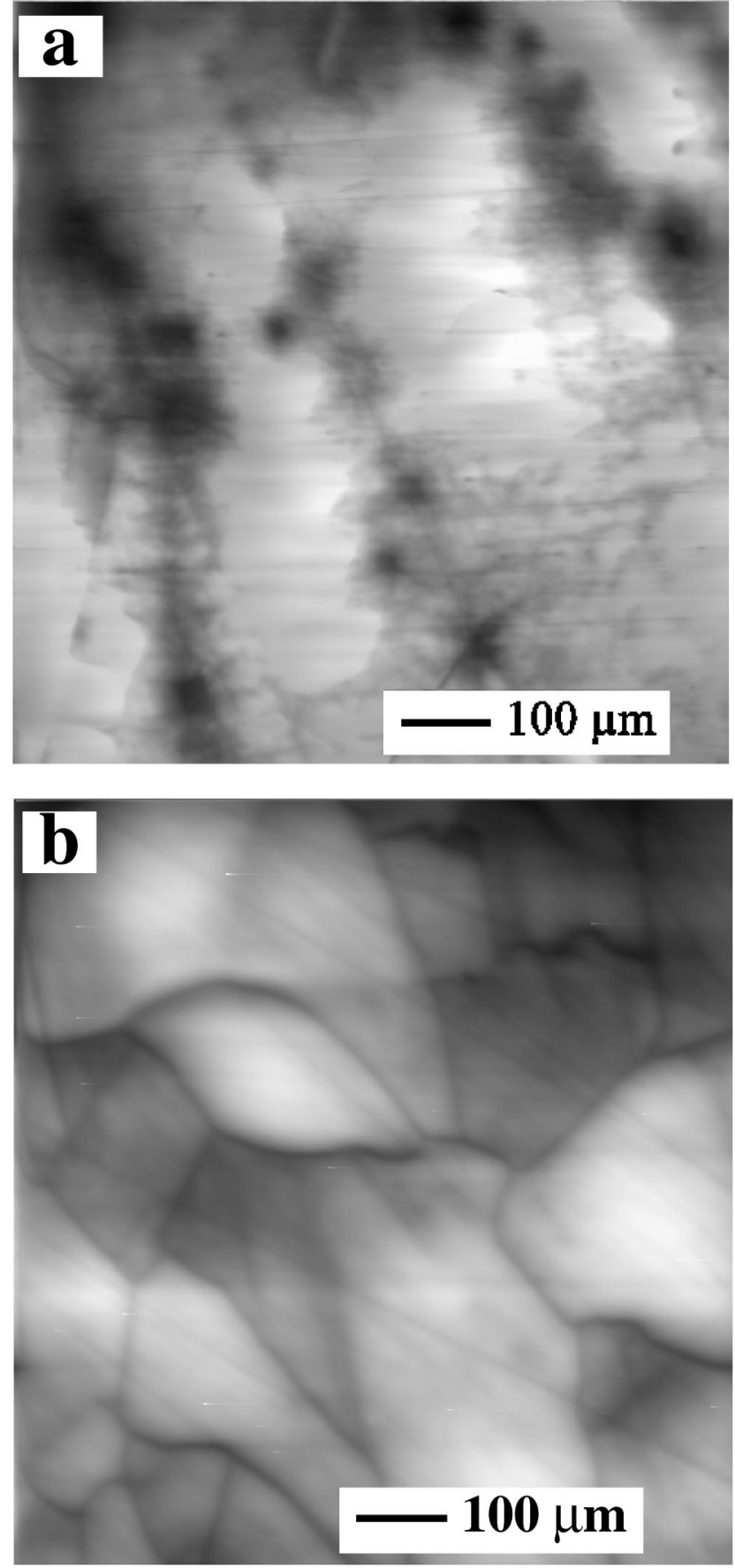

FIG. 3. CL micrographs obtained from GaSb:Cr crystals: (a) sample $\mathrm{Cr} 1$ and (b) sample $\mathrm{Cr} 4$.

and a uniform appearance in the interiors of the grains. ${ }^{7}$ As the concentration of $\mathrm{Cr}$ increases in the crystal, precipitates are observed whose number also increases from sample $\mathrm{Cr} 4$ to sample Cr1, see Fig. 3 .

\section{B. GaSb:V}

Figure 4 shows the CL spectra of the $\mathrm{V}$ doped samples labeled V1, V3, and V4. In these spectra, an evolution of the emission bands with the same trend as observed in the $\mathrm{Cr}$ doped samples can be seen. The band A is almost nonexistent in samples V1 and V2, the V-rich samples, while in

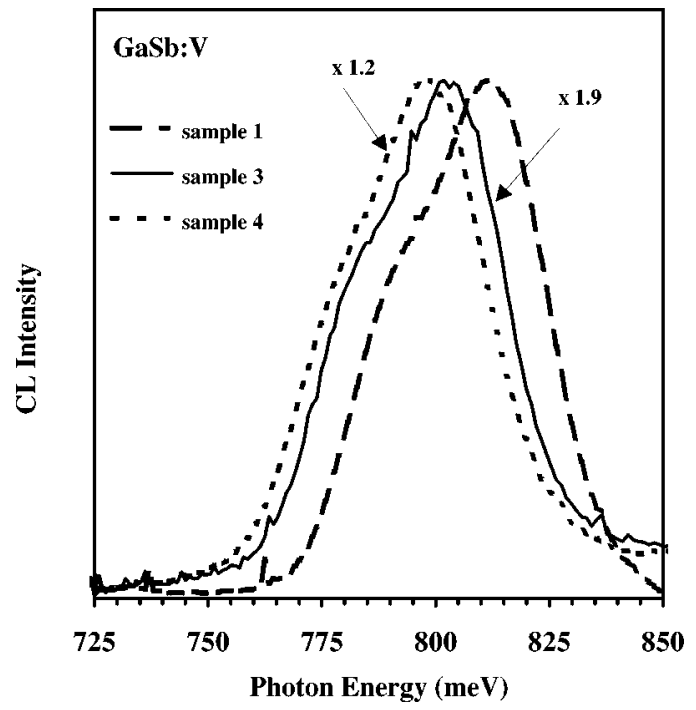

FIG. 4. CL spectra of V-doped GaSb from the different position in the ingot.

sample V4, corresponding to the vanadium deficient end of the ingot, band $\mathrm{A}$ is dominant. Therefore the degree of compensation of native defects, as in the Cr-doped samples, depends on the position in the ingot. In GaSb:V, the peak positions of both CL bands shift to higher energy with increasing $\mathrm{V}$ doping (see Table I). This shift may be due to impurity band effect in these degenerately doped crystals.

CL images of the vanadium-doped samples exhibit a more complex appearance than the CL images chromium-doped samples, but with some common features. Most of the crystal surface appears well smooth and featureless in the secondary electron images. The corresponding CL images are shown in as Fig. 5(a) with dark features at points on the subgrain boundaries. Comparison among the different V-doped samples shows that the size and number of the dark features increase with increasing $\mathrm{V}$ doping, suggesting a segregation process at the boundaries.

In some areas of $\mathrm{V}$-doped $\mathrm{GaSb}$ it is possible to find topographic features in the secondary-electron image, such as those shown in Fig. 5(c), associated to dark contrast in the corresponding CL image [Fig. 5(b)]. This result indicates a low incorporation efficiency of $\mathrm{V}$ in GaSb. In fact, one can clearly see the $\mathrm{V}$ inclusions in the crystal. X-ray microanalysis of those regions shows that the protrusions are vanadium rich and, to a lesser extent, antimony rich areas which indicates that they correspond to precipitates with vanadium as the main component. X-ray microanalysis data reveal an atomic percentage of the different elements in the precipitates of $24 \% \mathrm{Ga}, 51 \% \mathrm{Sb}$, and $24 \% \mathrm{~V}$. In precipitate free regions of the sample the $\mathrm{V}$ atomic percentage is too small to be observed by $\mathrm{x}$-ray micoanalysis.

\section{GaSb:Ru}

CL spectra from samples Ru1 to Ru3 reveal a luminescence band centered at $767 \mathrm{meV}$, which has not been previously reported to the best of our knowledge (Fig. 6). This band is quite intense in sample Ru1, is weaker in samples $\mathrm{Ru} 2$ and Ru3, and finally is not present in sample Ru4. This band exhibits a different temperature dependence than the 

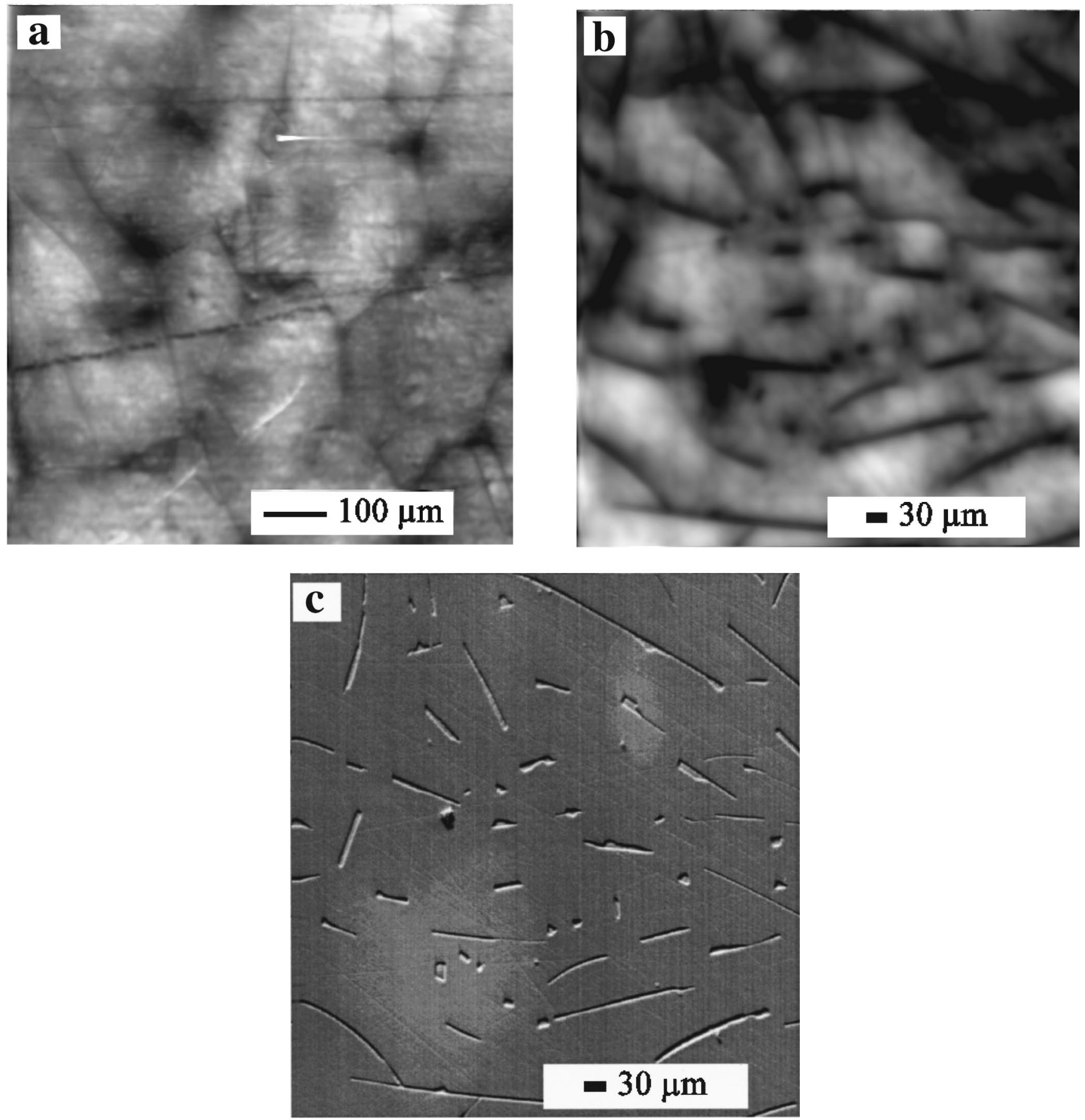

FIG. 5. CL and secondary-electron images of GaSb:V. (a) Representative CL image of the sample V2, (b) CL image of the region containing V-rich features in sample V3, and (c) secondary-electron image corresponding to the same area in sample V3.

other CL bands. Its peak shifts to lower energy as the temperature increases, and the magnitude of the shift is higher than the shift of band A. The fact that the $767 \mathrm{meV}$ band is strongest in sample Ru1 suggests that this band arises from ruthenium, which has the highest concentration in sample $\mathrm{Ru} 1$. It is interesting to note that at low levels of $\mathrm{Ru}$, the 777 meV peak (band A) totally disappears. Thus, the way in which $\mathrm{Ru}$ affects the native acceptor centers in $\mathrm{GaSb}$ is different from the previous studied dopants $(\mathrm{Cr}, \mathrm{V})$; $\mathrm{Ru}$ is more effective at reducing the native acceptors in $\mathrm{GaSb}$ than $\mathrm{Cr}$ or $\mathrm{V}$.
In the $\mathrm{CL}$ images from samples $\mathrm{Ru} 2$ to $\mathrm{Ru} 4$ a large number of subgrains of small size (around $100 \mu \mathrm{m}$ ) have been detected in the centers of the disks, whereas bigger and more uniform grains are observed near the edges of the samples. Also near the edges, dark spots, with CL contrast typical of dislocations or precipitates, appear inside the grains. In samples Ru2 and Ru3, a decoration effect of the dark spots is observed [Fig. 7(a)], similar to the often reported haloes surrounding dislocations in CL images of semiconductors. ${ }^{13}$ In sample $\mathrm{Ru} 1$, the $\mathrm{CL}$ contrast reveals an alignment of dark spots following preferred directions [Fig. 7(b)] similar to the 


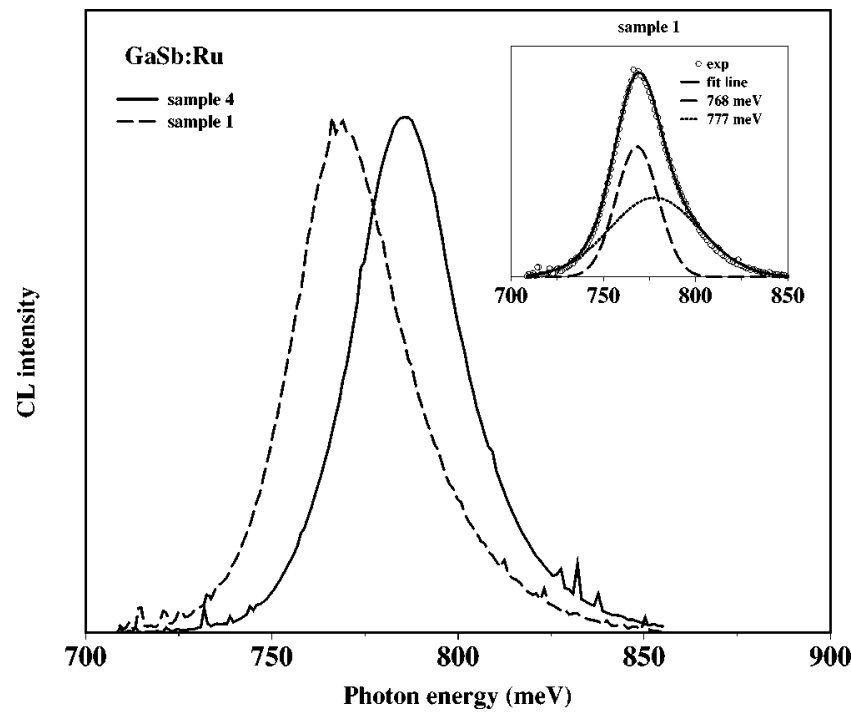

FIG. 6. CL spectra of GaSb:Ru samples. The deconvolution in two bands of the spectrum from sample Rul evidences a band at $767 \mathrm{meV}$ as is shown in the inset.

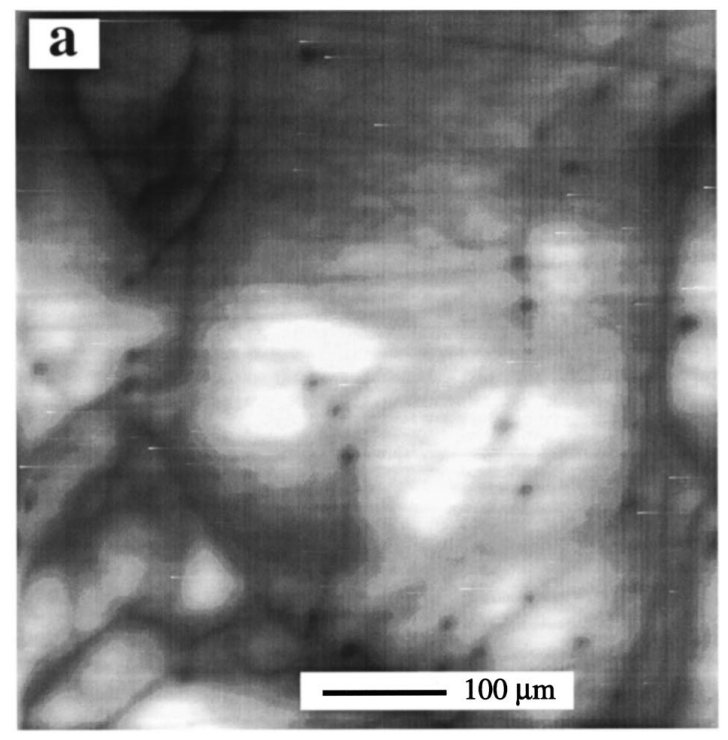

typical contrast of gliding bands in other materials. ${ }^{13}$ On the other hand, secondary electron images show that the shape of the precipitates in Ru-doped samples is quite different than in V-doped ones [Fig. 7(c)]. It is possible to distinguish rounded shapes and elongated precipitates. The concentration of these precipitates is highest in sample Rul and decreases from samples $\mathrm{Ru} 2$ to $\mathrm{Ru} 4$. The measured precipitate concentrations range from $5 \times 10^{4} \mathrm{~cm}^{-2}$ in sample Ru4, to $1 \times 10^{6} \mathrm{~cm}^{-2}$ in sample Ru1. CL images clearly reveal an inhomogeneous distribution in number, shape and size of the precipitates depending on the position along the ingot axis.

$\mathrm{X}$-ray microanalysis data confirm the formation of precipitates, preferentially in sample Ru1, as also occurs in the $\mathrm{Cr}$-doped and V-doped samples. However, the X-ray data reveal that the atomic percentage at the precipitates in the $\mathrm{Ru}-$ doped samples is $56 \% \mathrm{Ga}, 33 \% \mathrm{Sb}$, and $10 \% \mathrm{Ru}$. These samples have an excess of $\mathrm{Ga}$. This excess has been found previously in the literature ${ }^{14}$ to enhance the band A emission (Ga-rich material) and on the contrary, Sb-rich stoichiometry leads to a suppression of band A. In our samples, band A is only present in sample Ru1 competing with the band. We believe that the Ruthenium atom has too big a radius to be
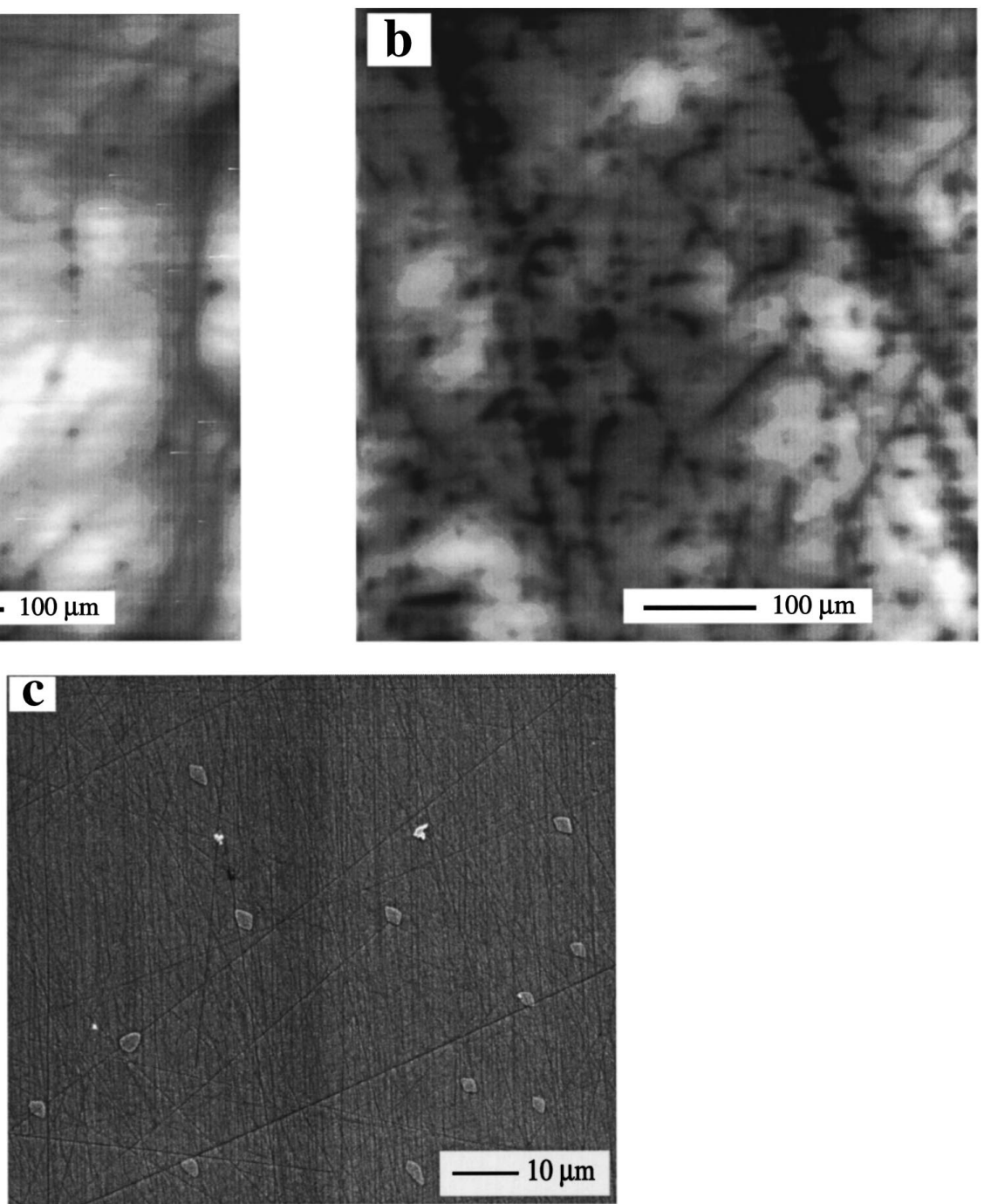

FIG. 7. CL and secondary-electron images of GaSb:Ru crystals. (a) CL image of sample Ru3, (b) CL image of sample Ru1, and (c) secondary-electron image of precipitates in sample $\mathrm{Ru} 2$. 
located at Ga sites, unlike $\mathrm{Cr}$ or $\mathrm{V}$ atoms, and is thus located at $\mathrm{Sb}$ sites in the lattice leading to a $\mathrm{Sb}$ deficient matrix.

It should be noted that any precipitate formation means that the solubility limit has been exceeded. Hence even though the concentration of $\mathrm{Ru}$ in sample Rul is higher, the effective incorporation in the matrix (on lattice sites) is much less. This explains the fact that the $777 \mathrm{meV}$ band (band A) is still observed in sample Ru1. Any change in luminescence spectra due to impurity doping depends on the incorporation of the dopant impurity on lattice sites. Precipitate formation reduces the effect of the impurity on the matrix.

From the present $\mathrm{CL}$ and $\mathrm{x}$-ray microanalysis investigations, we conclude that the best method to reduce the native acceptors is with low Ru-doping levels as in sample Ru4, in contrast to the Cr- or V-doped samples where the most effective suppresion of native defects occurs in the impurityrich end of the ingot (samples Cr1 and V1). To quantify the above statement, it would be necessary to determine the solubility limits of these impurities and their absolute values for segregation, which is not present in the literature to the best of our knowledge.

\section{CONCLUDING REMARKS}

Reduction of native acceptors was accomplished by transition-metal-doping in GaSb. In particular, three metals, namely, $\mathrm{Cr}, \mathrm{V}$, and $\mathrm{Ru}$, were chosen for technological reasons. The efficiency of impurity incorporation in the lattice was studied by CL spectroscopy and imaging, and by $\mathrm{x}$-ray microanalysis. Impurity segregation coefficients higher than unity for the elements $\mathrm{Cr}, \mathrm{V}$, and $\mathrm{Ru}$ in $\mathrm{GaSb}$ were observed. Further chemical analysis would be required to conclusively determine the absolute values of the segregation coefficients. The complete disappearance of the $777 \mathrm{meV}$ band in $\mathrm{GaSb}: \mathrm{Ru}$ at low Ru-doping levels was seen. These results are very interesting for potential device applications of GaSb. The appearance of a band at $767 \mathrm{meV}$ in $\mathrm{GaSb}: \mathrm{Ru}$ requires further investigation to get a better understanding of the interaction between $\mathrm{Ru}$ and the native defects in $\mathrm{GaSb}$.

\section{ACKNOWLEDGMENTS}

This work was supported by the DGICYT (Project No. PB93-1256) and CICYT (Project No. 95-0086-OP).
${ }^{1}$ A. M. Hennel, in Imperfections in III/V Materials, edited by R. K. Willardson, A. C. Beer, and E. R. Weber, Vol. 38 of Semiconductors and Semimetals (Academic, New York, 1993).

${ }^{2}$ E. M. Omel'yanovskii and V. I. Fistul, Transition Metal Impurities in Semiconductors (Adam Hilger, Bristol, 1986).

${ }^{3}$ B. Stepanek, P. Hubik, J. J. Mares, J. Kristofik, V. Sestakova, L. Pekarek, and J. Sestak, Semicond. Sci. Technol. 9, 1138 (1994).

${ }^{4}$ T. Adhikari and S. Basu, Mater. Sci. Eng. B 27, 47 (1994).

${ }^{5}$ P. S. Dutta, H. L. Bhat, and V. Kumar, J. Appl. Phys. 81, 5821 (1997).

${ }^{6}$ G. N. Panin, P. S. Dutta, J. Piqueras, and E. Dieguez, Appl. Phys. Lett. 67, 3584 (1995).

${ }^{7}$ B. Méndez, J. Piqueras, P. S. Dutta, and E. Dieguez, Appl. Phys. Lett. 67, 2648 (1995).
${ }^{8}$ B. Méndez and J. Piqueras, J. Appl. Phys. 69, 2776 (1991).

${ }^{9}$ M. Lee, D. J. Nicholas, K. E. Singer, and B. Hamilton, J. Appl. Phys. 59, 2895 (1986).

${ }^{10}$ C. Anayama, T. Tanahashi, H. Kuwatsuka, S. Nishiyama, S. Isozumi, and K. Nakajima, Appl. Phys. Lett. 56, 239 (1990).

${ }^{11}$ B. Méndez, J. Piqueras, P. S. Dutta, and E. Dieguez, Mater. Sci. Eng. B 42, 38 (1996).

${ }^{12}$ E. I. Georgitsé, L. M. Gutsulyak, V. I. Ivanov-Omskil, V. A. Smirnov, and Sh. U. Yuldashev, Sov. Phys. Semicond. 25, 1180 (1991).

${ }^{13}$ B. G. Yacobi and D. B. Holt, Cathodoluminescence Microscopy of Inorganic Solids (Plenum, New York, 1990).

${ }^{14}$ M. C. Wu and C. C. Chen, J. Appl. Phys. 72, 4275 (1992). 